\title{
Bulk Higgs and Gauge fields in a multiply warped braneworld model
}

\author{
Ashmita Das 1 , R. S. Hundi2 and Soumitra SenGupta 3 \\ Department of Theoretical Physics, \\ Indian Association for the Cultivation of Science, \\ 2A \& 2B Raja S.C. Mullick Road, \\ Kolkata - 700 032, India.
}

\begin{abstract}
We readdress the problems associated with bulk Higgs and the gauge fields in a 5-dimensional Randall-Sundrum model by extending the model to six dimensions with double warping along the two extra spatial dimensions. In this 6-dimensional model we have a freedom of two moduli scales as against one modulus in the 5dimensional model. With a little hierarchy between these moduli we can obtain the right magnitude for $W$ and $Z$ boson masses from the Kaluza-Klein modes of massive bulk gauge fields where the spontaneous symmetry breaking is triggered by bulk Higgs . We also have determined the gauge couplings of the standard model fermions with Kaluza-Klein modes of the gauge fields. Unlike the case of 5-dimensional model with a massless bulk gauge field, here we have shown that the gauge couplings and the masses of the Kaluza-Klein gauge fields satisfy the precision electroweak constraints and also obey the Tevatron bounds.
\end{abstract}

\footnotetext{
${ }^{1}$ E-mail address: tpad@iacs.res.in

${ }^{2}$ E-mail address: tprsh@iacs.res.in

${ }^{3}$ E-mail address: tpssg@iacs.res.in
} 


\section{Introduction}

The hierarchy between electroweak and Planck scales can be addressed in extra dimensional models. Among these, the model proposed by Randall and Sundrum (RS) assumes warp geometry of the space-time in 5 dimensions [1]. The fifth dimension has Planck scale length $r_{c}$ and is compactified on the space $S^{1} / Z_{2}$. Two 3-branes are supported on either side of this fifth dimension. The exponential suppression along the fifth dimension naturally suppresses Planck scale quantities of one 3-brane into electroweak scale on the second 3-brane, which is identified as TeV-brane and can be interpreted as our universe. In the original RS model the standard model fields are assumed to lie on the TeV-brane while only gravity propagates in the bulk. Later works have explored the phenomenology of bulk standard model fields in warped geometry model [2, 3, 4, 5, 6]. It was however shown that the models with bulk gauge and Higgs fields where the spontaneous symmetry breaking takes place in the bulk, encounters serious problems. The two main problems are:

(i) The non-Abelian gauge fields acquire masses through the Higgs vacuum expectation value (vev) generated through spontaneous symmetry breaking in the bulk. This vev being a bulk parameter has a magnitude of the order of the Planck scale and therefore lends a very large bulk mass $\sim$ Planck mass to the gauge boson in the bulk. As a result the lowest lying masses in the KK tower of the gauge boson on the visible brane becomes $\mathrm{TeV}$ which fails to comply with the $W$ and $Z$ boson masses $\leq 100 \mathrm{GeV}$. If one tries to reduce it by adjusting the bulk parameters then that would jeopardize the unique feature of Planck scale to TeV scale warping, i.e. the resolution of gauge hierarchy problem which was the original motivation of such a warped geometry model.

(ii) For an Abelian gauge boson with zero bulk mass, the massless Kaluza-Klein (KK) mode on the $\mathrm{TeV}$-brane corresponds to photon. However, the first excited state in the KK tower has an unacceptably large coupling with fermions. This puts a stringent bound on the mass of this state such that the model may survive the direct search bound at the Fermilab Tevatron as well as precision electroweak constraints. However, the mass of this first KK mode turns out to be much lower than the above bound. Once again it is impossible either to reduce the coupling or to increase the mass by adjusting the bulk parameters without disturbing the resolution of the gauge hierarchy/fine tuning problem.

We refer our readers to [3, 6, 7, 8, 9, 10] where both these problems have been discussed in details. Recently, in a generalized 5-dimensional RS model with non-flat visible brane, 
by adjusting the brane cosmological constant the problem coming from the precision electroweak tests have been averted and also the bulk Higgs problem has been resolved [7. The brane cosmological constant however was found to be negative implying that the visible brane in such a case is an anti-de Sitter 3-brane.

In the present work we address these problems from a different viewpoint i.e. in the backdrop of a 6-dimensional doubly warped model with flat 3-branes which is an extension of the original RS model to more than one extra dimensions[11]. In this 6-dimensional model two extra spatial coordinates are compactified such that the space-time manifold is $\left[M^{(1,3)} \times S^{1} / Z_{2}\right] \times S^{1} / Z_{2}$. Compared to the RS model, here the two extra dimensions, denoted by angular coordinates $y, z$, are doubly warped. Four 4-branes are located at the orbifolded points: $y=0, \pi, z=0, \pi$. The intersection of any two 4-branes gives a 3-brane. The 3-brane located at $(y, z)=(\pi, 0)$ is identified with our universe. Analogous to the $\mathrm{RS}$ setup, the mass scale suppression can be felt along both the coordinates $y, z$. We can choose the moduli of these coordinates, say $R_{y}$ and $r_{z}$, such that $\mathrm{TeV}$ scale masses can be generated on the visible brane located at $(y, z)=(\pi, 0)$. Since there is an extra freedom through an additional modulus in this model compared to the 5-dimensional RS model, we explore if the above problems relating to bulk Higgs can be solved in the 6-dimensional doubly warped model by adjusting the moduli $R_{y}, r_{z}$ suitably.

We organize our paper as follows. In the following section we explain some essential features of the 6-dimensional doubly warped model. In Sec. 3 we describe the KK mode analysis of gauge bosons and fermions in 6-dimensional bulk and the corresponding modes on the visible 3-brane. In Sec. 4 we present our results and argue that the precision electroweak tests put no additional constraints on this model. We conclude in Sec. 5.

\section{The 6-dimensional doubly warped model}

As explained previously, the 6-dimensional doubly warped model has space-time of six dimensions and the extra two spatial dimensions are orbifolded by $Z_{2}$ symmetry [11. The manifold under consideration is $\left[M^{(1,3)} \times S^{1} / Z_{2}\right] \times S^{1} / Z_{2}$ with four non-compact dimensions denoted by $x^{\mu}, \mu=0, \cdots, 3$. Since we are interested in doubly warped model, the metric in this model can be chosen as

$$
d s^{2}=b^{2}(z)\left[a^{2}(y) \eta_{\mu \nu} d x^{\mu} d x^{\nu}+R_{y}^{2} d y^{2}\right]+r_{z}^{2} d z^{2}
$$

As explained before the angular coordinates $y, z$ represent the extra spatial dimensions with moduli $R_{y}, r_{z}$, respectively. The Minkowski matrix in the usual 4-dimensions has 
the form $\eta_{\mu \nu}=\operatorname{diag}(-1,1,1,1)$. The functions $a(y), b(z)$ give warp factors in the $y$ and $z$ directions, respectively. The total bulk-brane action of this model has a form [11]

$$
\begin{aligned}
S & =S_{6}+S_{5} \\
S_{6} & =\int d^{4} x d y d z \sqrt{-g_{6}}\left(R_{6}-\Lambda\right), \\
S_{5} & =\int d^{4} x d y d z\left[V_{1} \delta(y)+V_{2} \delta(y-\pi)\right]+\int d^{4} x d y d z\left[V_{3} \delta(z)+V_{4} \delta(z-\pi)\right]
\end{aligned}
$$

Here, $V_{1,2}$ and $V_{3,4}$ are brane tensions of the branes located at $y=0, \pi$ and $z=0, \pi$, respectively. $\Lambda$ is the cosmological constant in 6 -dimensions. The 3 -branes are located at the intersection points of the four 4-branes.

After solving Einstein's equations the solutions to the warp functions of the metric as given in eq. (1) have a form [11]

$$
\begin{aligned}
a(y) & =\exp (-c|y|), \quad b(z)=\frac{\cosh (k z)}{\cosh (k \pi)} \\
c & \equiv \frac{R_{y} k}{r_{z} \cosh (k \pi)}, \quad k \equiv r_{z} \sqrt{\frac{-\Lambda}{10 M_{P}^{4}}}
\end{aligned}
$$

Here, $M_{P}$ is the Planck scale. The warp factors $a(y)$ and $b(z)$ give largest suppression from $y=0, z=\pi$ brane to $y=\pi z=0$ brane. For this reason we can interpret the 3-brane formed out of the intersection of 4-branes at $y=\pi$ and $z=0$ as our standard model brane. The suppression factor $f$ on the standard model brane can be written as

$$
f=\frac{\exp (-c \pi)}{\cosh (k \pi)}
$$

The desired suppression of $10^{-16}$ on the standard model brane can be obtained for different combinations of the parameters $c$ and $k$. However, from the relation for $c$ in eq. (3) it can be noticed that in order not to have large hierarchy in the moduli $R_{y}$ and $r_{z}$, either of $c$ or $k$ must be large where as other is small, e.g. $c \sim 11.4$ and $k \sim 0.1$. This implies that the warping along $y$ is large whereas that along $z$ is small. It has been argued that this feature may offer an explanation of the small mass hierarchy among the standard model fermions [1].

The 6-dimensional model which has been described here is thus viable in explaining the hierarchy between Planck scale and the electroweak scale without introducing large hierarchy between the moduli $R_{y}$ and $r_{z}$. In this model KK modes of bulk scalar fields have been studied [12]. Bulk fermion fields have also been studied in this model with a possibility of localizing them on a 4-brane [13]. However the possibility of bulk gauge and 
Higgs field in this model has not been explored yet. In the following section we derive the KK modes of the gauge field, fermion fields and the corresponding couplings to estimate the viability of this model in respect to the problems discussed earlier. We reiterate that our aim is to explore if we can put the Higgs in the bulk of such 6-dimensional multiply warped model without invoking any contradiction with the precision electroweak test [3, 6 ] as was encountered in the 5-dimensional RS model.

\section{Gauge bosons and fermions in the bulk}

In this section we explain the KK decomposition and eigenvalue equations of KK gauge bosons and KK fermions which arise from the respective bulk fields after integrating over the two extra dimensions of the model.

\subsection{KK modes of the gauge bosons}

For simplicity, we consider a $\mathrm{U}(1)$ gauge theory, but our derivation given below is applicable to non-Abelian theory as well. In a realistic model the gauge fields can acquire non-zero masses due to spontaneous symmetry breaking. In our model, Higgs mechanism can take place in the bulk of the 6-dimensions and the vev of the Higgs field will be of the order of Planck scale. The vev of the Higgs field contributes to generate the bulk mass for the gauge field. Hence, after spontaneous symmetry breaking the invariant action can be written as

$$
S_{G}=\int d^{4} x d y d z \sqrt{-G}\left(-\frac{1}{4} G^{M K} G^{N L} F_{K L} F_{M N}-\frac{1}{2} M^{2} G^{M K} A_{M} A_{K}\right),
$$

where, $M$ is the bulk mass $\sim M_{P}$ and $G=\operatorname{det}\left(G_{A B}\right)$ is the determinant of the metric $G_{A B}$ which is given in eq. (11). $F_{K L}=\partial_{K} A_{L}-\partial_{L} A_{K}$ is the gauge field strength. Exploiting the gauge symmetry we can choose the gauge where $A_{4}=A_{5}=0$. The KK decomposition of the gauge field can be taken as

$$
A_{\mu}=\sum_{n, p} A_{\mu}^{(n, p)}(x) \xi_{n}(y) \chi_{p}(z) / \sqrt{R_{y} r_{z}}
$$

The KK fields in the 4-dimensions $A_{\mu}^{(n, p)}$ carry two indices $n, p$ due to the two additional dimensions of the model. The functions $\xi_{n}(y)$ and $\chi_{p}(z)$ give KK wave functions in the $y$ and $z$ directions, respectively. Substituting the above KK decomposition in eq. (5) 
and integrating over the $y$ and $z$ coordinates, we demand that the resulting action in the 4-dimensions must have a form

$$
\sum_{n, p}-\frac{1}{4} F_{\mu \nu}^{(n, p)} F^{(n, p) \mu \nu}-\frac{1}{2} m_{n, p}^{2} A_{\mu}^{(n, p)} A^{(n, p) \mu},
$$

where $m_{n, p}$ is the mass of the KK field $A_{\mu}^{(n, p)}$. This can be achieved provided the KK wave functions satisfy the following orthonormality condition.

$$
\int d y \xi_{n}(y) \xi_{n^{\prime}}(y)=\delta_{n n^{\prime}}, \quad \int d z b(z) \chi_{p}(z) \chi_{p^{\prime}}(z)=\delta_{p p^{\prime}}
$$

Moreover, in addition to the above normalization conditions the following eigenvalue equations for the $\xi_{n}$ and $\chi_{p}$ must also be satisfied:

$$
\begin{aligned}
\frac{1}{R_{y}^{2}} \partial_{y}\left(a^{2} \partial_{y} \xi_{n}\right)-m_{p}^{2} a^{2} \xi_{n} & =-m_{n, p}^{2} \xi_{n} \\
\frac{1}{r_{z}^{2}} \partial_{z}\left(b^{3} \partial_{z} \chi_{p}\right)-M^{2} b^{3} \chi_{p} & =-m_{p}^{2} \chi_{p} .
\end{aligned}
$$

Here, $m_{p}$ is a mass parameter which is determined by solving the equation for $\chi_{p}(z)$, and the value of $m_{p}$ determines the KK mass $m_{n, p}$ through the eigenvalue equation for $\xi_{n}$, as given above.

The second of the eq. (9) can be solved by approximating $b(z) \sim \exp [-k(\pi-z)]=$ $\exp [-k \tilde{z}]$. By writing $\tilde{\chi}_{p}(z)=\exp (-3 k \tilde{z} / 2) \chi_{p}(z)$ the eigenvalue equation for $\tilde{\chi}_{p}$ takes the form,

$$
z_{p}^{2} \frac{d^{2} \tilde{\chi}_{p}}{d z_{p}^{2}}+z_{p} \frac{d \tilde{\chi}_{p}}{d z_{p}}+\left(z_{p}^{2}-\nu_{p}^{2}\right) \tilde{\chi}_{p}=0,
$$

where $z_{p}=\frac{m_{p}}{k^{\prime}} \exp (k \tilde{z})$ and $\nu_{p}^{2}=\frac{9}{4}+\left(\frac{M}{k^{\prime}}\right)^{2}$. Here, $k^{\prime}=k / r_{z}$. The solutions to the above equation are Bessel functions of order $\nu_{p}$, and we can write

$$
\chi_{p}(z)=\frac{1}{N_{p}} \exp \left(\frac{3}{2} k \tilde{z}\right)\left[J_{\nu_{p}}\left(z_{p}\right)+b_{p} Y_{\nu_{p}}\left(z_{p}\right)\right]
$$

where, $N_{P}$ and $b_{p}$ are some constants. By demanding that the function $\chi_{p}(z)$ be continuous at the orbifold fixed points $z=0, \pi$ we get the following approximate solution which determines the spectrum for $m_{p}$.

$$
3 J_{\nu_{p}}\left(x_{\nu_{p}}\right)+x_{\nu_{p}}\left(J_{\nu_{p}-1}\left(x_{\nu_{p}}\right)-J_{\nu_{p}+1}\left(x_{\nu_{p}}\right)\right)=0
$$

where $x_{\nu_{p}}=\frac{m_{p}}{k^{\prime}} \exp (k \pi)$. After solving for $m_{p}$ using the above equation, we can compute the KK mass $m_{n, p}$ by solving the first of eq. (9)). By writing $\tilde{\xi}_{n}=\exp (-c|y|) \xi_{n}$, the eigenvalue equation for $\tilde{\xi}_{n}(y)$ becomes,

$$
y_{n}^{2} \frac{d^{2} \tilde{\xi}_{n}}{d y_{n}^{2}}+y_{n} \frac{d \tilde{\xi}_{n}}{d y_{n}}+\left(y_{n}^{2}-\nu_{n}^{2}\right) \tilde{\xi}_{n}=0
$$


where $y_{n}=\frac{m_{n, p}}{k^{\prime}} \exp (c|y|) \cosh (k \pi)$ and $\nu_{n}^{2}=1+\left(\frac{m_{p}}{k^{\prime}}\right)^{2} \cosh ^{2}(k \pi)$. The solution for $\xi_{n}(y)$ can be written in terms of Bessel function of order $\nu_{n}$ multiplied by growing exponential factor as

$$
\xi_{n}(y)=\frac{1}{N_{n}} \exp (c|y|)\left[J_{\nu_{n}}\left(y_{n}\right)+b_{n} Y_{\nu_{n}}\left(z_{n}\right)\right]
$$

where, $N_{n}$ and $b_{n}$ are some constants. Again, by demanding that the function $\xi_{n}(y)$ be continuous at the orbifold fixed points $y=0, \pi$ the following equation determines the KK mass $m_{n, p}$.

$$
J_{\nu_{n}}\left(x_{\nu_{n}}\right)+x_{\nu_{n}}\left(J_{\nu_{n}-1}\left(x_{\nu_{n}}\right)-J_{\nu_{n}+1}\left(x_{\nu_{n}}\right)\right) / 2=0
$$

where,

$$
x_{\nu_{n}}=\frac{m_{n, p}}{k^{\prime}} \exp (c \pi) \cosh (k \pi)
$$

The actual KK mass of a gauge field is found by first solving the eq. (12) for $m_{p}$ and then solving the eq. (15), which is described in the previous paragraph. The wave function of these KK gauge fields is product of wave functions given in eqs. (11) and (14). In the above analysis if we put the bulk gauge boson mass $M=0$, we easily obtain the various KK mode solutions and the corresponding masses. In this case the lowest lying KK mode is massless which corresponds to the standard model photon.

A nice feature of the KK gauge fields in the 6-dimensional doubly warped model is that their wave functions can be decomposed into product of functions in the two extra dimensions, a feature which may not be evident for the bulk fermion fields which is the subject of the next subsection.

\subsection{KK modes of the fermions}

The invariant action for a bulk fermion field $\Psi$ in 6-dimensions is [3, 5, 6]

$$
S_{f}=\int d^{4} x d y d z \sqrt{-G}\left\{E_{a}^{A}\left[\frac{i}{2}\left(\bar{\Psi} \Gamma^{a} \partial_{A} \Psi-\partial_{A} \bar{\Psi} \Gamma^{a} \Psi\right)+\frac{\omega_{b c A}}{8} \bar{\Psi}\left\{\Gamma^{a}, \sigma^{b c}\right\} \Psi\right]-M_{f} \bar{\Psi} \Psi\right\}
$$

Here, the capital letter $A$ denotes index in the curved space and the lower case letters $a, b, c$ denote indices in the tangent space. $\omega_{b c A}$ is spin connection and $E_{a}^{A}$ is inverse vielbein. $M_{f}$ is the bulk mass and $\sigma^{b c}=\frac{i}{2}\left[\Gamma^{b}, \Gamma^{c}\right]$. The Dirac matrices $\Gamma^{a}$ in 6 -dimensions would be $8 \times 8$, and they can be taken as [14]:

$$
\Gamma^{\mu}=\gamma^{\mu} \otimes \sigma^{0}, \quad \Gamma^{4}=i \gamma_{5} \otimes \sigma^{1}, \quad \Gamma^{5}=i \gamma_{5} \otimes \sigma^{2}
$$

Here, $\gamma^{\mu}$ are the Dirac matrices in 4-dimensions and $\gamma_{5}=i \gamma^{0} \gamma^{1} \gamma^{2} \gamma^{3} . \sigma^{i}, i=1,2,3$, are the Pauli matrices and $\sigma^{0}$ is the $2 \times 2$ unit matrix. The chirality in 6 -dimensions is defined 
by the matrix $\bar{\Gamma}=\Gamma^{0} \Gamma^{1} \Gamma^{2} \Gamma^{3} \Gamma^{4} \Gamma^{5}$ as $\bar{\Gamma} \Psi_{ \pm}= \pm \Psi_{ \pm}$. The chiral fermions in 6-dimensions have both left- and right-handed chirality of 4-dimensions, which can be projected by the operators $P_{L, R}=\left(1 \mp i \Gamma^{0} \Gamma^{1} \Gamma^{2} \Gamma^{3}\right) / 2$.

As explained in Sec. 1, we are interested in estimating the gauge coupling of standard model fermions to the KK gauge bosons. We take the bulk mass of the fermions $M_{f}$ to be zero, since the masses of standard model fermions are much below the Planck scale. The term that is associated with the spin connection in eq. (17) would give no contribution, since the metric in eq. (1) is diagonal. Hence, in our particular case of interest we expand the first term of eq. (17), which has the following form:

$$
\begin{aligned}
S_{f}=\int d^{4} x d y d z & \left\{b^{4} a^{3} R_{y} r_{z} i\left(\bar{\Psi}_{+L} \Gamma^{\mu} \partial_{\mu} \Psi_{+L}+\bar{\Psi}_{+R} \Gamma^{\mu} \partial_{\mu} \Psi_{+R}+\bar{\Psi}_{-L} \Gamma^{\mu} \partial_{\mu} \Psi_{-L}+\bar{\Psi}_{-R} \Gamma^{\mu} \partial_{\mu} \Psi_{-R}\right)\right. \\
& +\left[\bar{\Psi}_{+L}\left(\Gamma^{4} D_{y}+\Gamma^{5} D_{z}\right) \Psi_{+R}+\bar{\Psi}_{+R}\left(\Gamma^{4} D_{y}+\Gamma^{5} D_{z}\right) \Psi_{+L}\right. \\
& \left.\left.+\bar{\Psi}_{-L}\left(\Gamma^{4} D_{y}+\Gamma^{5} D_{z}\right) \Psi_{-R}+\bar{\Psi}_{-R}\left(\Gamma^{4} D_{y}+\Gamma^{5} D_{z}\right) \Psi_{-L}\right]\right\}
\end{aligned}
$$

where the differential operators are defined as: $D_{y}=\frac{i}{2} b^{4} r_{z}\left(a^{4} \partial_{y}+\partial_{y} a^{4}\right)$ and $D_{z}=$ $\frac{i}{2} a^{4} R_{y}\left(b^{5} \partial_{z}+\partial_{z} b^{5}\right)$. In the subscript of the fields $\Psi$ the \pm indicates the chirality in 6 dimensions and the $L, R$ stands for the left- and right-handed chirality of the 4-dimensions. Terms in line 2 and 3 of the above equation give effective masses for the KK modes in the 4-dimensions. These terms indicate that in general we cannot decompose the wave functions into $y$ and $z$ parts separately, like what we have done for the KK wave functions of the gauge bosons as described previously in eq. (6) . Hence, for the bulk fermions the KK decomposition can be taken as

$$
\begin{aligned}
& \Psi_{+L,-R}\left(x^{\mu}, y, z\right)=\frac{1}{\sqrt{R_{y} r_{z}}} \sum_{j, k} \psi_{+L,-R}^{(j, k)}\left(x^{\mu}\right) f_{+L,-R}^{(j, k)}(y, z) \otimes\left(\begin{array}{l}
1 \\
0
\end{array}\right), \\
& \Psi_{-L,+R}\left(x^{\mu}, y, z\right)=\frac{1}{\sqrt{R_{y} r_{z}}} \sum_{j, k} \psi_{-L,+R}^{(j, k)}\left(x^{\mu}\right) f_{-L,+R}^{(j, k)}(y, z) \otimes\left(\begin{array}{l}
0 \\
1
\end{array}\right) .
\end{aligned}
$$

In the above equation various fields of the form $\psi^{(j, k)}\left(x^{\mu}\right)$ are the KK fields living in the 4-dimensions and $f$ 's are the KK wave functions depending on both $y$ and $z$ coordinates. Substituting the above KK decomposition into eq. (19) and integrating over the $y$ and $z$ we can get the action of the form

$$
\begin{aligned}
S_{f}=\int d^{4} x & \sum_{j, k} \bar{\psi}_{+L}^{(j, k)} i \gamma^{\mu} \partial \psi_{+L}^{(j, k)}+\bar{\psi}_{+R}^{(j, k)} i \gamma^{\mu} \partial \psi_{+R}^{(j, k)}+\bar{\psi}_{-L}^{(j, k)} i \gamma^{\mu} \partial \psi_{-L}^{(j, k)}+\bar{\psi}_{-R}^{(j, k)} i \gamma^{\mu} \partial \psi_{-R}^{(j, k)} \\
& -M_{j, k}\left(\bar{\psi}_{+L}^{(j . k)} \psi_{+R}^{(j, k)}+\bar{\psi}_{+R}^{(j . k)} \psi_{+L}^{(j, k)}+\bar{\psi}_{-L}^{(j . k)} \psi_{-R}^{(j, k)}+\bar{\psi}_{-R}^{(j . k)} \psi_{-L}^{(j, k)}\right)
\end{aligned}
$$


provided the following normalization and the eigenvalue equations for the KK wave functions are satisfied:

$$
\begin{aligned}
& \int d y d z b^{4}(z) a^{3}(y)\left(f_{+R,+L,-R,-L}^{(j, k)}(y, z)\right)^{*} f_{+R,+L,-R,-L}^{\left(j^{\prime}, k^{\prime}\right)}(y, z)=\delta^{j, j^{\prime}} \delta^{k, k^{\prime}} \\
&\left(i \mathcal{D}_{y}+\mathcal{D}_{z}\right) f_{+R}^{(j, k)}(y, z)=-M_{j, k} f_{+L}^{(j, k)}(y, z) \\
&\left(-i \mathcal{D}_{y}+\mathcal{D}_{z}\right) f_{+L}^{(j, k)}(y, z)=-M_{j, k} f_{+R}^{(j, k)}(y, z) \\
&\left(i \mathcal{D}_{y}+\mathcal{D}_{z}\right) f_{-L}^{(j, k)}(y, z)=M_{j, k} f_{-R}^{(j, k)}(y, z) \\
&\left(-i \mathcal{D}_{y}+\mathcal{D}_{z}\right) f_{-R}^{(j, k)}(y, z)=M_{j, k} f_{-L}^{(j, k)}(y, z)
\end{aligned}
$$

where the differential operators are: $\mathcal{D}_{y}=\frac{i}{2 R_{y}}\left(4 \partial_{y} a+2 a \partial_{y}\right)$ and $\mathcal{D}_{z}=\frac{i}{2 r_{z}} a\left(5 \partial_{z} b+2 b \partial_{z}\right)$. Here, $M_{j, k}$ is the mass of the KK fermion $\psi^{(j, k)}$.

As explained previously, we are interested in standard model fermion coupling with the KK modes of gauge field. The zero mode of the KK fermions are identified with the standard model fermions. The wave function for these fields can be solved from eq. (23) by putting $M_{j, k}=0$. Here, we show the solution for the wave function $f_{+R}^{(0,0)}(y, z)$ and the solutions for other chiral fermions can be analogously worked out. The eigenvalue equation we are interested in is

$$
\left(i \mathcal{D}_{y}+\mathcal{D}_{z}\right) f_{+R}^{(0,0)}(y, z)=0
$$

where the differential operators $\mathcal{D}_{y}$ and $\mathcal{D}_{z}$ are defined below eq. (23). For the zero-mode case we can write the function $f_{+R}^{(0,0)}$ as a product of $y$ and $z$ parts, say $f_{+R}^{(0,0)}(y, z)=$ $f_{y}(y) f_{z}(z)$. This simplification happens only for the zero-mode case, since the factor $a(y)$ in the operators $\mathcal{D}_{y, z}$ can be taken out and the right-hand side of the above equation is zero. Substituting this form of $f_{+R}^{(0,0)}(y, z)$ in the above equation we get

$$
-\frac{1}{R_{y}} \frac{\left(-4 c+2 \partial_{y}\right) f_{y}}{f_{y}}+i \frac{1}{r_{z}} \frac{\left(5 \partial_{z} b+2 b \partial_{z}\right) f_{z}}{f_{z}}=0
$$

Since the functional dependence on $y$ and $z$ are completely separated out, we can solve for $f_{y}$ and $f_{z}$ by taking $\frac{\left(-4 c+2 \partial_{y}\right) f_{y}}{f_{y}}=c_{1}$, where $c_{1}$ is a separation constant. In terms of $c_{1}$ the functional dependences of $f_{y}$ and $f_{z}$ are given below

$$
f_{y}(y)=\exp \left(\frac{1}{2}\left(c_{1}+4 c\right) y\right), \quad f_{z}(z)=\frac{\exp \left(\frac{-i c_{1} r_{z}}{k R_{y}} \tan ^{-1}(\tanh (k z / 2)) \cosh (k \pi)\right)}{\cosh ^{5 / 2}(k z)}
$$

The value of $c_{1}$ can be worked out in terms of $c$ and $k$ by normalizing the wave function $f_{+R}^{(0,0)}(y, z)$ using eq. (22) 


\section{Bulk phenomenology}

In the previous section we have given a description of the KK modes of the gauge bosons and fermions in the bulk of a 6-dimensional doubly warped model. Now using the mode expansion for the bulk fields, we can calculate the gauge couplings of standard model fermions with the KK modes of the gauge bosons.

We now address the two problems mentioned in the beginning. Recall that in 5dimensional RS model it is found that for non-zero bulk mass for non-Abelian gauge field the lowest lying mode has mass much higher than $100 \mathrm{GeV}$ i.e. the masses for $W$ and $Z$ bosons. Also, for the massless gauge boson the gauge coupling with the first exited KK gauge boson is larger than one and hence the standard model fermions are strongly coupled [3, 6]. Due to this a stringent lower bound of $\sim 10 \mathrm{TeV}$ on the mass of the first exited KK boson arose because of the precision electroweak tests.

In this section we repeat this exercise in the 6-dimensional doubly warped model, and will show that due to the presence of an additional modulus we can tune the lowest KK mode mass for non-Abelian gauge field near $100 \mathrm{GeV}$ although the spontaneous symmetry breaking takes place in the bulk with a bulk Higgs field with vev $\sim$ Planck scale. Furthermore, for a gauge boson with a zero bulk mass the coupling to mass ratio of the first excited KK mode can survive the precision electroweak test without putting any additional restriction on the model.

The action between the bulk fermions and gauge bosons can be written as [ $[3,6]$

$$
S_{\mathrm{int}}=\int d^{4} x d y d z \sqrt{-G} g_{6 d} \bar{\Psi}\left(x^{\mu}, y, z\right) i \Gamma^{a} E_{a}^{A} A_{A}\left(x^{\mu}, y, z\right), \Psi\left(x^{\mu}, y, z\right)
$$

where $g_{6 d}$ is the gauge coupling in the 6-dimensions as has been discussed in Sec. 3 . Substituting the KK decomposition for the gauge and fermion fields in the above equation and also reminding that we are working in the gauge choice where $A_{4}=A_{5}=0$, we get the gauge coupling in the 4-dimensions as

$$
g_{+R}^{(j, k)(n, p)}=\int d y d z g_{0} \pi b^{4} a^{3}\left(f_{+R}^{(j, k)}(y, z)\right)^{*} f_{+R}^{(j, k)}(y, z) \xi_{n}(y) \chi_{p}(z)
$$

where $g_{0}=g_{6 d} / \sqrt{\pi R_{y} \pi r_{z}}$ is the effective 4-dimensional gauge coupling. In the above equation we have given gauge coupling for the KK fermion $\psi_{+R}^{(j, k)}$ with the KK gauge field $A_{\mu}^{(n, p)}$. Similarly, the gauge couplings with the other KK fermions can be easily obtained by replacing the + with - and $R$ with $L$ accordingly in the above equation. The KK wave functions: $f$ 's, $\xi$ and $\chi$, in the above equation should be the normalized wave functions as given by eqs. (8) and (22). However, in our particular case of interest, where we 


\begin{tabular}{||c|c|c||c|c|c||}
\hline \multicolumn{3}{||c||}{$\frac{M}{k^{\prime}}=0.5$} & \multicolumn{3}{c||}{$\frac{M}{k^{\prime}}=1.0$} \\
$m_{1,2}=143.78$ & $m_{1,3}=194.15$ & $m_{1,4}=244.52$ & $m_{1,2}=148.33$ & $m_{1,3}=199.21$ & $m_{1,4}=249.58$ \\
$\tilde{g}^{1,2}=0.0015$ & $\tilde{g}^{1,3}=0.0028$ & $\tilde{g}^{1,4}=0.0009$ & $\tilde{g}^{1,2}=0.0006$ & $\tilde{g}^{1,3}=0.0027$ & $\tilde{g}^{1,4}=0.0011$ \\
\hline$m_{2,1}=180.23$ & $m_{2,2}=237.94$ & $m_{2,3}=295.40$ & $m_{2,1}=184.53$ & $m_{2,2}=243.25$ & $m_{2,3}=300.97$ \\
$\tilde{g}^{2,1}=0.0127$ & $\tilde{g}^{2,2}=0.0012$ & $\tilde{g}^{2,3}=0.0023$ & $\tilde{g}^{2,1}=0.0124$ & $\tilde{g}^{2,2}=0.0005$ & $\tilde{g}^{2,3}=0.0022$ \\
\hline$m_{3,1}=261.73$ & $m_{3,2}=322.73$ & $m_{3,3}=383.48$ & $m_{3,1}=266.29$ & $m_{3,2}=328.30$ & $m_{3,3}=389.56$ \\
$\tilde{g}^{3,1}=0.0106$ & $\tilde{g}^{3,2}=0.0010$ & $\tilde{g}^{3,3}=0.0019$ & $\tilde{g}^{3,1}=0.0104$ & $\tilde{g}^{3,2}=0.0004$ & $\tilde{g}^{3,3}=0.0019$ \\
\hline
\end{tabular}

Table 1: The gauge couplings $g^{(0,0)(i, j)}$ of the standard model fermions are given in the form $\tilde{g}^{i, j}=\frac{g^{(0,0)(i, j)}}{g_{0}}$, where $g_{0}$ is the effective 4-dimensional gauge coupling. The masses of the KK gauge bosons $m_{i, j}$ are given in $\mathrm{GeV}$ units. $M$ is the bulk gauge boson mass and $k^{\prime}=k / r_{z}$. The non-zero values for $\frac{M}{k^{\prime}}$ are indicated in the table. $\frac{1}{r_{z}}=7 \times 10^{17} \mathrm{GeV}, k=$ 0.25 and $c=11.52$. The lowest lying mode $m_{1,1}$, which corresponds to $W$ or $Z$ boson, is not included in the table.

are interested in precision electroweak tests, we compute gauge couplings of the standard model fermions with the KK gauge fields. Hence, the wave functions for the fermions are of the form in eq. (26) and the corresponding functions for the KK gauge fields are given in eqs. (11) and (14).

Now, in order to compute the gauge couplings the unknown parameters that need to be fixed are $k, c, r_{z}$ and the bulk mass of the gauge fields $M$. The non-zero value for the bulk gauge mass $M$ is around the Planck scale. We can determine the remaining parameters by making the following demands: (a) the lowest non-zero mass of the KK tower of the bulk gauge boson should be identified with either $W$ or $Z$ boson mass, (b) the suppression $f$ of eq. (4) should be $\sim 10^{-16}$ and (c) the hierarchy between the moduli $R_{y}$ and $r_{z}$ should not be too large. The expression for the KK gauge boson mass is given in eq. (16). For the lowest non-zero KK gauge boson mass which is identifie as $m_{1,1}$ the root $x_{\nu_{n}}$ would be $\mathcal{O}(1)$. The factor $\exp (c \pi) \cosh (k \pi)$ in this equation, which is the inverse of $f$, should be $\sim 10^{16}$. By demanding that the lowest KK mode $m_{1,1}$ has mass of $\sim 100 \mathrm{GeV}$, from eq. (16) we can naively estimate that $k^{\prime} \sim 10^{17} \mathrm{GeV}$. Since we would argue that $k \sim 0.1$, a consistent value for the scale $r_{z}$ is $\frac{1}{r_{z}}=7 \times 10^{17} \mathrm{GeV}$, which is about 14 times smaller than the Planck scale. The parameters $k$ and $c$ can be determined from the fact that we should not get large hierarchy between the moduli $R_{y}$ and $r_{z}$ and also we should get the desired suppression of $f \sim 10^{-16}$ on the standard model brane. We have estimated that for $k=0.25$ and $c=11.52$, the ratio between the 


\begin{tabular}{||c|c|c||}
\hline \multicolumn{3}{||c||}{$\frac{M}{k^{\prime}}=0$} \\
\hline$m_{1,1}=93.15$ & $m_{1,2}=142.0$ & $m_{1,3}=192.38$ \\
$\tilde{g}^{1,1}=0.0168$ & $\tilde{g}^{1,2}=0.0019$ & $\tilde{g}^{1,3}=0.0028$ \\
\hline$m_{2,1}=178.45$ & $m_{2,2}=235.91$ & $m_{2,3}=293.12$ \\
$\tilde{g}^{2,1}=0.0128$ & $\tilde{g}^{2,2}=0.0015$ & $\tilde{g}^{2,3}=0.0023$ \\
\hline$m_{3,1}=259.96$ & $m_{3,2}=320.71$ & $m_{3,3}=381.21$ \\
$\tilde{g}^{3,1}=0.0107$ & $\tilde{g}^{3,2}=0.0012$ & $\tilde{g}^{3,3}=0.0020$ \\
\hline
\end{tabular}

Table 2: The gauge couplings $g^{(0,0)(i, j)}$ of the standard model fermions are given in the form $\tilde{g}^{i, j}=\frac{g^{(0,0)(i, j)}}{g_{0}}$, where $g_{0}$ is the effective 4-dimensional gauge coupling. The masses of the KK gauge bosons $m_{i, j}$ are given in GeV units. $M$ is the bulk gauge boson mass, which is taken to be zero and $k^{\prime}=k / r_{z}$. The values of $k, c$ and $r_{z}$ in this case are same as that in Table 1. The lowest lying mode $m_{0,0}$, which corresponds to photon, is not included in the table.

moduli is $\frac{R_{y}}{r_{z}}=61$, which is not unacceptably large, and also the suppression $f$ came out to be $1.45 \times 10^{-16}$. For these particular values of $k, c$ and $1 / r_{z}$ we have given the gauge couplings and the corresponding masses of the excited KK gauge fields in Table 1. In this table the gauge couplings $g^{(i, j)}$, where $i, j$ are integers, of the standard model fermion are given as a fraction of the 4-dimensional coupling $g_{0}$. In the case of $\frac{M}{k^{\prime}}=0.5$ or $=1.0$, we have found that the lowest non-zero mode has mass of about $95 \mathrm{GeV}$. Hence this mode can be identified with the $W$ or $Z$ gauge boson. Since we have got the right amount of $W, Z$ boson masses for the above described values of $k, c, \frac{1}{r_{z}}$, we use the same set of values to get the gauge couplings and KK gauge boson masses in the case where the bulk mass $M$ is zero. In this case we have found that the lowest mode $m_{0,0}$ has zero mass which can be identified with the photon state. The non-zero KK masses of the photon field and their corresponding gauge coupling values are given in Table 2.

As stated in Sec. 1 that the 5-dimensional RS model suffers from the precision electroweak tests due to the fact that the first excited KK gague boson has coupling larger than one with the standard model field. To parameterize the precision electroweak constraints in extra dimensional models the following quantity has been defined [3, 15].

$$
V=\sum_{n}\left(\frac{g_{n}}{g_{0}} \frac{m_{W}}{M_{n}}\right)^{2} .
$$

Here, $m_{W}$ is the mass of the $W$ gauge boson and $M_{n}$ is the higher KK gauge boson mass. The summation on $n$ in the above equation is over all the higher KK gauge masses $M_{n}$ 
with corresponding gauge couplings $g_{n}$. In our 6 -dimensional model the index $n$ would be replaced by a pair of integers and we should sum over all non-zero higher order modes. It has been shown that by fitting to the precision electroweak observables the quantity $V$ should satisfy the condition: $V<0.0013$ at $95 \%$ confidence level [3]. It can be easily checked that this bound can be respected by the gauge couplings and the KK masses of the tables 1 and 2. From both these tables, we can notice that the gauge couplings are decreasing with increasing the KK gauge masses for a particular value of $\frac{M}{k^{\prime}}$. Hence, in the summation of eq. (29) only the first few higher KK modes are relevant. We have checked that for $\frac{M}{k^{\prime}}=0.5, V$ has come out to be about $5 \times 10^{-5}$. In the case of photon where bulk mass is zero the value of $V$ is found out to be about 0.000257 . From these results we can conclude that precision electroweak tests can be satisfied in the 6-dimensional doubly warped model without introducing too much hierarchy in the moduli $R_{y}$ and $r_{z}$.

At the Tevatron the higher KK gauge bosons have been searched in the channel $P \bar{P} \rightarrow$ $X \rightarrow e^{+} e^{-}$and a limit of $M_{T}>700 \mathrm{GeV}$ on the heavy vector gauge boson $(X)$ mass has been put-in [16]. In our case the gauge couplings of the higher KK modes have been reduced from the 4-dimensional coupling $g_{0}$ by some factors which are given in tables 1 and 2. Hence, in our case, the Tevatron bounds for the non-zero KK mode masses should be greater than $700 \times \tilde{g}^{i, j} \mathrm{GeV}$. As an example, the $\mathrm{KK}$ mode of $93.15 \mathrm{GeV}$ mass of the Table 2 has the gauge coupling ratio of 0.0168 . Hence, the lower bound from the Tevatron on this KK mode mass would be about $12 \mathrm{GeV}$, which is much lower than our calculated value of $93.15 \mathrm{GeV}$. Like wise, from each column of the tables 1 and 2 it can be easily seen that the above mentioned Tevatron bounds can be satisfied. So the 6-dimensional warped model is not only free from the precision electroweak constraints but also from the Tevatron limits.

\section{Conclusions}

The extra dimensional phenomenological models in a warped geometry encounters problems in putting the Higgs and the gauge fields in the bulk. It was shown that it is impossible to construct proper $W$ and $Z$ boson masses on the brane from the KK modes of a non-Abelian bulk gauge field through spontaneous symmetry breaking in the bulk. Also proper coupling and masses for the first KK excitation of a massless bulk gauge field consistent with electroweak precision test as well as Fermilab Tevatron mass bound is hard to obtain without changing the bulk parameter of the theory from their desired values. In this work we have shown that it is possible to resolve both these problems in 
a multiply warped geometry model where there are more than one modulus. Considering a 6-dimensional model we have shown that by setting one of the modulus approximately two orders smaller than the Planck scale, we can have the the mass for the lowest lying mode of the bulk gauge field ( with bulk mass $\sim M_{P}$, acquired through a spontaneous symmetry breaking in the bulk) on the TeV-brane to be of the order of $100 \mathrm{GeV}$ which therefore may be identified with the $W, Z$ boson mass. Moreover, such a choice for the moduli which does not contradict the main spirit of the RS model lowers the coupling of the first KK mode excitation of a massless bulk gauge field so that it can escape the electroweak precision test. We have determined the KK mode masses as well as their couplings for different choices of the parameter of the theory namely the ratio of the bulk mass and the bulk cosmological constant. In the entire analysis the value of the warp factor is maintained at $10^{-16}$ so that the resolution of the gauge hierarchy problem, the main objective of these models can be achieved. These findings can be easily extended to models with even larger number of warped extra dimensions [?]. One would then arrive at similar conclusions with a lesser hierarchy among different moduli. We can therefore conclude that a consistent description of bulk Higgs and gauge field with spontaneous symmetry breaking in the bulk can be obtained in a warped geometry model if the RS model in 5-dimensions is generalized to six or higher dimensions with more than one moduli. The phenomenology of these models therefore becomes an interesting area of study for the forthcoming collider experiments.

\section{References}

[1] L. Randall and R. Sundrum, Phys. Rev. Lett. 83, 3370 (1999); ibid 83, 4690 (1999).

[2] W.D. Goldberger and M.B. Wise, Phys. Rev. D 60, 107505 (1999).

[3] H. Davoudiasl, J.L. Hewett and T.G. Rizzo, Phys. Lett. B 473, 43 (2000); H. Davoudiasl, J.L. Hewett and T.G. Rizzo, Phys. Rev. D 63, 075004 (2001).

[4] A. Pomarol, Phys. Lett. B 486, 153 (2000); T. Gherghetta and A. Pomarol, Nucl. Phys. B 586, 141 (2000).

[5] Y. Grossman and M. Neubert, Phys. Lett. B 474, 361 (2000).

[6] S. Chang, J. Hisano, H. Nakano, N. Okada and M. Yamaguchi, Phys. Rev. D 62, 084025 (2000). 
[7] P. Dey, B. Mukhopadhyaya and S. SenGupta, Phys. Rev. D 81, 036011 (2010)

[8] C.H. Chang and J.N. Ng, Phys. Lett. B 488, 390 (2000); S.J. Huber and Q. Shafi, Phys. Rev. D 63, 045010 (2001).

[9] H. Davoudiasl, J.L. Hewett, B. Lillie and T.G. Rizzo, Phys. Rev. D 70, 015006 (2004); H. Davoudiasl, B. Lillie and T.G. Rizzo, JHEP 0608, 042 (2006).

[10] K. Agashe, A. Delgado, M.J. May and R. Sundrum, JHEP 0308, 050 (2003); Y. Hosotani and M. Mabe, Phys. Lett. B 615, 257 (2005); Y. Hosotani, S. Noda, Y. Sakamura and S. Shimasaki, Phys. Rev. D 73, 096006 (2006); M.S. Carena, E. Ponton, J. Santiago and C.E.M. Wagner, Nucl. Phys. B 759, 202 (2006); A.D. Medina, N.R. Shah and C.E.M. Wagner, Phys. Rev. D 76, 095010 (2007); A. Djouadi and G. Moreau, Phys. Lett. B 660, 67 (2008); M. Bauer, Acta Phys. Polon. Supp. 3, 131 (2010) [ arXiv:0910.4876 [hep-ph]]; M.E. Albrecht, M. Blanke, A.J. Buras, B. Duling and K. Gemmler, JHEP 0909, 064 (2009). .

[11] D. Choudhury and S. SenGupta, Phys. Rev. D 76, 064030 (2007).

[12] R. Koley, J. Mitra and S. SenGupta, Europhys. Lett. 91, 31001 (2010).

[13] R. Koley, J. Mitra and S. SenGupta, Phys. Rev. D 78, 045005 (2008).

[14] B.A. Dobrescu and E. Ponton, JHEP 0403, 071 (2004); G. Burdman, B.A. Dobrescu and E. Ponton, JHEP 0602, 033 (2006).

[15] T.G. Rizzo and J.D. Wells, Phys. Rev. D 61, 016007 (1999).

[16] C. Amsler et al. (Particle Data Group), Phys. Lett. B667, 1 (2008); T. Altonen et al., Phys. Rev. D 78, 012008 (2008); T. Altonen et al., Phys. Rev. Lett. 102, 031801 (2009). 